\title{
Diagnostic Value of 64-Slice Multi Detector Computed Tomographic Angiography in Assessment of Complicated Coronary Artery Bypass Graft
}

\author{
EISHA R. MOHAMED, M.D.*; IBRAHIM M. HELMY, M.D.**; LOBNA Kh. SAKR, M.D.* and \\ KHALED A. EL-GHAMRAWY, M.Sc.*** \\ The Department of Radiodiagnosis, Faculty of Medicine for Girls, Al-Azhar University, Cairo*, \\ International Medical Center, National Heart Institute, Cairo and Al-Ahrar Teaching Hospital, Zagazig** and \\ Al-Ahrar Teaching Hospital, Zagazig***, Egypt
}

\begin{abstract}
Background: Coronary Artery Bypass Grafting (CABG) is to replace occluded or stenosed coronary arteries with patent venous or arterial conduits. Long-term sequelae after CABG include native coronary artery disease progression and de novo arthrosclerosis in grafts, resulting in recurrent ischemic symptoms and mortality.
\end{abstract}

Aim of Study: This study was undertaken to form the basis of review on the role of 64 Multi-Detector Computed Tomography (MDCT) in detecting possible complications in post coronary artery graft $(\mathrm{CABG})$ patients.

Material and Methods: During the period from November 2015 to August 2017, a prospective evaluation of 50 patients with suspected post-CABG complications underwent MDCT coronary angiography in radiology department of International Medical Center in Egypt. Informed consent was obtained from all patients and the study had institutional review board approval. Descriptive statistics were used to analyze the data.

Results: There were 40 males and 10 females, their mean age \pm (SD) of $59 \pm 3.9$ years. A total of 129 grafts were available for evaluation. From the total 129 graft 25 grafts were occluded as 24 of them showing the occlusion at the proximal anastomosis and the remaining one at the distal anastomosis. 8 grafts showed significant stenosis. Two showing ulceration at anastomotic site and 6 showing kinking course while one was showing competitive flow distally and one showing retrograde felling. Four grafts show stent placement. From the total evaluated 50 patients four patients were underwent redu operation. One patient had partial thrombosis in left subclavian artery encroaching upon LIMA origin. Three patients were suffering from fever one had pneumonia and the other two had chest wall infection with retrosternal extension and associated mediastinal involvement. Four patients show sternal dehiscence. One patient showed localized pericardial hematoma. Sex patients showed ascending aortic aneurysm and two

Correspondence to: Dr. Eisha Ramadan Mohamed,

The Department of Radiodiagnosis, Faculty of Medicine for Girls, Al-Azhar University, Cairo, Egypt showed main pulmonary artery aneurysm. All these complications occurred from 6 menthes to 13 years post CABG surgery.

Conclusion: MDCT is an easy non-invasive technique which showed to be effective in diagnosis of post-operative $\mathrm{CABG}$ related and non-related complications and is very informitive in difficult clinical situations.

Key Words: MDCT (Multidetector Computed Tomography) - Coronary Artery Bypass Graft (CABG).

\section{Introduction}

THE aim of coronary artery bypass grafting (CA$\mathrm{BG}$ ) is to replace occluded or stenosed coronary arteries with patent venous or arterial conduits. Long-term sequelae after CABG include native coronary artery disease progression and de novo arthrosclerosis in grafts, resulting in recurrent ischemic symptoms and mortality [1]. Complications of Coronary Artery Bypass Grafts (CABGs): Graft thrombosis/stenosis, malposition or kinking and spasm. Iatrogenic complications of graft (retained clips, graft aneurysm and pseudoaneurism, ascending aorta dissecting aneurysm and pseudoaneurism, ostium stenosis and occlusion of the coronary arteries after Bentall procedure) [2] . Non graft related surgical complications: Pleural/ pericardial effusion, sternal/mediastinal infection and pulmonary embolism [3]. In recent years, Computed Tomography Coronary Angiography (CTCA) underwent substantial improvements, especially electrocardiographic (ECG)-gated CTCA [4]. ECGgated MDCT provides a fast and reliable, noninvasive method for assessing patients after Coronary Artery Bypass Graft (CABG). However, prob- 
lems may occur in patients with arrhythmia and dense calcifications in native coronary arteries with subsequent difficulties in the assessment of vessel stenosis [5]. Sensitivity and specificity for assessment of graft patency ranged between 85$100 \%$ and $95-100 \%$, whereas sensitivity and specificity for detection of $>50 \%$ diameter stenosis of CABG ranged between $75-100 \%$ and $89-100 \%$, respectively [5]. CTCA is very needful in imaging patients for re-do cardiac surgery to reduce the incidence for CABG-injury in re-sternotomy [6] The aim of our present study is to form the basis of a review on the role of 64-slice MDCT in detecting post $\mathrm{CABG}$ complications in our group of patients scanned at Radiology Department of International Medical Center in Egypt.

\section{Material and Methods}

During the period from November 2015 to August 2017, a total of 50 patients; 40 males and 10 females, their ages ranging from 41 to 85 years old with mean age \pm (SD) of $59 \pm 3.9$ years. All with suspected post-CABG complications underwent MDCT coronary angiography in the Radiology Department of International Medical Center in Egypt. Informed consent was obtained from all patients.

\section{Indications:}

To confirm or rule out complicated CABG in patients of previous CABG and suffering from symptoms and showing signs raise the suspicious of complications.

Referred from cardiology specialist from outpatient cardiology clinics either from cardiology clinic of the International Medical Center or other cardiology clinics which have referral contract with the hospital.

\section{Inclusion and exclusion criteria:}

The inclusion criteria included patients with post $\mathrm{CABG}$ operative need to assess the patency of their grafts, rule out any post operative complications, assess the progression of the disease in the native arteries and/or plan further surgical intervention. The exclusion criteria include Arrhythmias, heart rate above $80 / \mathrm{min}$ not responding to medical preparation, clinically unfit patients (unable to stop breathing during the examination or renal impairment) and/or allergy to iodinated contrast media.

\section{MDCT examination protocol:}

A combination of oral and intravenous betablockers has been used prior to the examination. Patients with heart rate $>70$ beat $/ \mathrm{min}$ were given cardio-selective beta-blocker; $100 \mathrm{mg}$ of Metoprolol or Atenolol orally 1 hour before the study to obtain a stable low heart rate, provided that contraindications to $\beta$-blockers are excluded. If heart rate was still $>70$ beat $/ \mathrm{min}$ the examination was postponed to another setting [6]. No adverse reactions to beta-blocker were recorded during our study. All examinations were performed by using MDCT Philips Ingenuity core 64 TM v3.5.7.25001 (Philips healthcare systems, Netherlands) with 64 detector rows. Monitoring of the electrocardiogram was performed continuously during the examination, and all image acquisitions were made during a single inspiratory breath-hold. An antero-posterior and lateral scout acquisitions from clavicle level to the diaphragmatic face of the heart were obtained in each patient to determine the position of the heart and define the scan volume for further imaging. The scan parameters are $64 \times 0.6$ collimation, 0.3 s rotation time, pitch of 0.32 , a section thickness of $0.5 \mathrm{~mm}$ and a rotation time of $0.4 \mathrm{sec}$. The tube current was $300+40 \mathrm{~mA}$ at $100-120 \mathrm{kV}$. Scanning direction; cranio-caudal. Mean scan time was 12 seconds \pm 1.5 , and total time for the examination was less than 10 minutes. For all examinations, $120 \mathrm{ml}$ of non-ionic contrast (Omnipaque $370 \mathrm{mg}$ Daiichi Pharmaceutical, Tokyo, Japan), was injected through an 18-gauge cannula positioned in the antecubital vein at a flow rate of $5.5 \mathrm{ml} / \mathrm{s}$. This injection was automatically followed by injection of about $40-50 \mathrm{cc}$ of saline at a flow rate of $5 \mathrm{ml} / \mathrm{sec}$ using a programmed dual head power injector pump (MedRad; USA) to maintain good opacification of the coronary vessels and the grafts with wash out of contrast material from the SVC and right side of the heart that may cause artifacts. In our cases with previous CABG operation; the scanning range must include the entire course of the arterial or venous grafts. No calcium scoring was done for our patients due to the presence of radiopaque surgical clips that would lead to false high scores. Then, automated determination of the starting time using the "Bolus-tracing technique" was determined in the lumen of the descending aorta using $30 \mathrm{ml}$ intravenous contrast agent at a flow rate of $5.5 \mathrm{ml} / \mathrm{s}$. The time interval between the bolus injection and the maximal enhancement was measured, and the starting time of the scan was calculated as 3 seconds after the transit time of contrast agent. For all patients, the standard built-in reconstruction algorithm was used for image reconstruction. The data sets were recon- 
structed with small Field of View (FOV) confined at the heart and with a slice thickness of $0.625 \mathrm{~mm}$ and $0.40 \mathrm{~mm}$ increments during the mid to-end diastolic phase, $65 \%$ to $75 \%$ of the R-R interval. A second dataset was reconstructed with a large FOV to include the entire chest in each cross section with a slice thickness of $5 \mathrm{~mm}$. All acquired data were transferred to a separate computer workstation (Advanced workstation 4.2, GE Healthcare, Milwaukee, USA) equipped with CardIQ software (GE Medical Systems). Depending on vessel morphology and quality of the MDCT data set, different post-processing techniques such as MaximumIntensity Projection (MIP), Curved Multiplanar Reconstruction (CMPR), and Volume Rendering (VR) were applied to assess the origin and course of the coronary vessels. High definition mode was used in all the scans. Gated coronary CT angiography software (Snap-shot Pulse, GE Health care, Milwaukee, USA) was used in 9 patients. Padding of the tube-on time was used to allow the reconstruction to adapt to minor heart rate variation. Patients were scanned with maximal padding of $200 \mathrm{~ms}$ in high-definition mode [7]. The CT examination was performed by an experienced radiologic technologist (M.K.). The interpretation of CT images was performed by a senior radiologist (I.M.H.) with an experience of over 18 years exclusively in cardiovascular imaging.

\section{Statistical analysis:}

Results were collated and entered into a database (Microsoft Excel 2010) detailing patient characteristics, patient complaints and graft complications. The data was analyzed using Statistical Package for Social Science (SPSS) Version 21 for windows. The patient characteristics, complaints and graft complications were analyzed using descriptive statistics documenting their number.

\section{Results}

There were 50 patients constituted the study group with a total 129 grafts. The 50 patients were 40 males and 10 females, their ages ranging from 41 to 85 years old with mean age \pm (SD) of $59 \pm 3.9$ years. Young study population is due to a multifactorial risk factors. Patients demographics are summarized in (Table 1), patients predisposing risk factors (Table 2) and patients complaints (Table 3). All patients had a heart rate of 60 to 70 beats/ minute at the time of scan acquisition. A total of 129 grafts were available for evaluation. There were 47 arterial and 82 venous grafts. Graft characteristics of these 50 patients are summarized in (Tables 4,5). Graft related complications regarding the 129 evaluated grafts are summarized in (Table 6) while the non-graft related complications of these 50 patients are summarized at (Table 7).

Twenty four patients had graft occlusion at proximal anastomosis Fig. (1) and one patient at distal anastomosis Fig. (2). One patient showed pleural effusion and pneumonia Fig. (3) diagnosed earlier by chest X-ray. One patient had pericardial hematoma which was missed by echocardiography Fig. (4). Two patients show chest wall infection by clinical examination, the diagnosis and extent (retrosternal extension and associated mediastinal involvement) of which was confirmed by CT Fig. (5). Four patients show non-union of previous sternotomy with evident sternal diastasis. One patient had partial thrombosis in left subclavian artery encroaching upon LIMA origin Fig. (6). There were incidental finding of aneurysmal dilatation of ascending aorta in four patients and main pulmonary artery in two patients.

Table (1): Patients demographics.

\begin{tabular}{ll}
\hline Item characteristic & \\
\hline - Number & 50 \\
- Gender females/males & $10 / 40$ \\
- Age group from & $41-85$ years old \\
- Age (years, Mean $\pm \mathrm{SD}$ ) & $59 \pm 3.9$ \\
- Time of symptoms post CABG (years, & $6 \pm 2$ \\
Mean $\pm \mathrm{SD}$ ) & \\
\hline
\end{tabular}

Table (2): Patients predisposing risk factors.

\begin{tabular}{lcc}
\hline \multirow{2}{*}{ Risk factors } & \multicolumn{2}{c}{ All studied patients } \\
\cline { 2 - 3 } & No. & $\%$ \\
\hline Diabetes mellitus & 39 & 78 \\
Hypertension & 42 & 84 \\
Smoking & 31 & 62 \\
Dyslipidemia & 33 & 66 \\
Obesity & 18 & 36 \\
Atherosclerosis & 21 & 42 \\
Previous myocardial infarction & 2 & 4 \\
\hline
\end{tabular}

Table (3): Patients complaints.

\begin{tabular}{lcc}
\hline \multirow{2}{*}{ Risk factors } & \multicolumn{2}{c}{ All studied patients } \\
& \multicolumn{2}{c}{$(\mathrm{N}=50)$} \\
\cline { 2 - 3 } & No. & $\%$ \\
\hline Chest pain & 48 & 96 \\
Respiratory distress & 21 & 42 \\
Fever & 3 & 6 \\
Dray cough & 2 & 4 \\
Productive cough & 1 & 2 \\
\hline
\end{tabular}


Table (4): Number of the grafts included in this study regarding their type, number, landing site/s and patency.

\begin{tabular}{|c|c|c|c|c|}
\hline & Patent & Stenosed & Occluded & Competitive flow \\
\hline $\begin{array}{l}\text { Arterial (47): } \\
\text { - In situ (45): } \\
\text { LIMA (42) }\end{array}$ & $\begin{array}{l}\text { LAD }: 34 \\
\text { OM : } 2 \\
\text { D } 1: 3\end{array}$ & LAD : 2 & OM then LAD : 1 & - \\
\hline RIMA (3) & RCA : 1 & & RCA : 1 & RCA : 1 \\
\hline $\begin{array}{c}\text { - Free (2): } \\
\text { RA (1) }\end{array}$ & $\mathrm{OM}: 1$ & - & - & - \\
\hline Free RIMA (1) & $\mathrm{D}: 1$ & - & - & - \\
\hline 47 & 42 ( 5 were kinked but patent) & 2 & 2 & 1 \\
\hline $\begin{array}{c}\text { Venous (82) } \\
\text { SVG (82) }\end{array}$ & $\begin{array}{l}\text { LAD }: 8 \\
\text { OM }: 16 \\
\text { HOM-LCX }: 1 \\
\text { RCA }: 6 \\
\text { RCA-LAD }: 1 \\
\text { RCA-PDA }: 2 \\
\text { D }: 10 \\
\text { LCX }: 1 \\
\text { PDA }: 7 \\
\text { PLB }: 1\end{array}$ & $\begin{array}{l}\text { OM : } 1 \\
\text { RCA : } 1 \\
\text { D : } 2 \\
\text { PDA : } 2\end{array}$ & $\begin{array}{l}\text { LAD : } 1 \\
\text { OM : } 1 \\
\text { RCA : } 1\end{array}$ & - \\
\hline $\begin{array}{r}129: \\
82\end{array}$ & 53 (1 was kinked) & 6 & $\begin{array}{l}\text { Unknown : } 20 \\
23\end{array}$ & - \\
\hline
\end{tabular}

Table (5): Graft types and numbers according to nature and anastomotic type.

\begin{tabular}{|c|c|c|c|c|c|}
\hline \multirow{3}{*}{ Graft types } & \multirow{2}{*}{\multicolumn{2}{|c|}{$\begin{array}{l}\text { All numbers of studied grafts } \\
\qquad(\mathrm{N}=129)\end{array}$}} & \multirow{2}{*}{$\begin{array}{l}\text { CABG related } \\
\text { complications }\end{array}$} & \multicolumn{2}{|c|}{$\begin{array}{l}\text { All studied CABG } \\
\qquad(\mathrm{N}=129)\end{array}$} \\
\hline & & & & No. & $\%$ \\
\hline & No. & $\%$ & Significantly narrowed & 8 & 6.2 \\
\hline \multirow[b]{2}{*}{ Total arterial grafts } & \multirow{2}{*}{47} & \multirow{2}{*}{36.4} & Ulceration & 2 & 1.6 \\
\hline & & & Kinking & 6 & 4.7 \\
\hline Single arterial & 46 & 35.6 & Competitive flow & 1 & 0.8 \\
\hline \multirow{2}{*}{ Sequential arterial } & \multirow{2}{*}{1} & \multirow{2}{*}{0.8} & Retrograde felling & 1 & 0.8 \\
\hline & & & Stent placement & 4 & 3.1 \\
\hline Total venous grafts & 82 & 63.6 & Redu operation & 4 & 3.1 \\
\hline \multirow{2}{*}{ Single venous } & 78 & 60.5 & Clip stenosis & 2 & 1.6 \\
\hline & 4 & 3.1 & Clip artifact & 2 & 1.6 \\
\hline & & & Calcifications & 2 & 1.6 \\
\hline
\end{tabular}

Table (6): CABG related complications.
Table (7): Non-graft related complications.

\begin{tabular}{lcc}
\hline \multirow{2}{*}{ Non-graft related complications } & \multicolumn{2}{c}{$\begin{array}{c}\text { All studied patients } \\
(\mathrm{N}=50)\end{array}$} \\
\cline { 2 - 3 } & No. & $\%$ \\
\hline Pleural effusion & 1 & 2 \\
Pneumonia & 1 & 2 \\
Pericardial hematoma & 1 & 2 \\
Sternal infection and collection & 2 & 3 \\
Sternal diastasis & 4 & 8 \\
Subclavian artery thrombosis & 1 & 2 \\
Ascending aorta aneurysm & 6 & 2 \\
Main pulmonary artery aneurysm & 2 & 4 \\
\hline
\end{tabular}



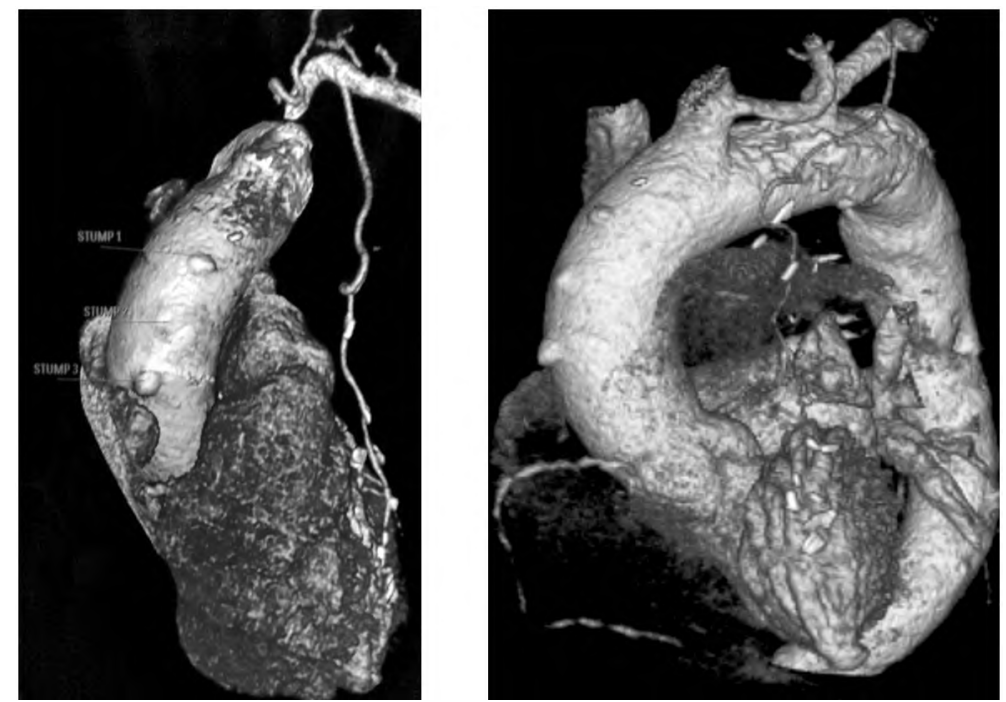

Fig. (1): 64 years old female patient, smoker, hypertensive and non-diabetic. In 2007, she developed recurrent attacks of chest pain followed by a bypass operation. Chest pain recurred 5 weeks ago. 3D VR showing Three star like projections at the anterior surface of the ascending aorta denoting occluded grafts as well as patent LIMA to LAD. The RCA shows significant stenosis at R2 with retrograde felling.
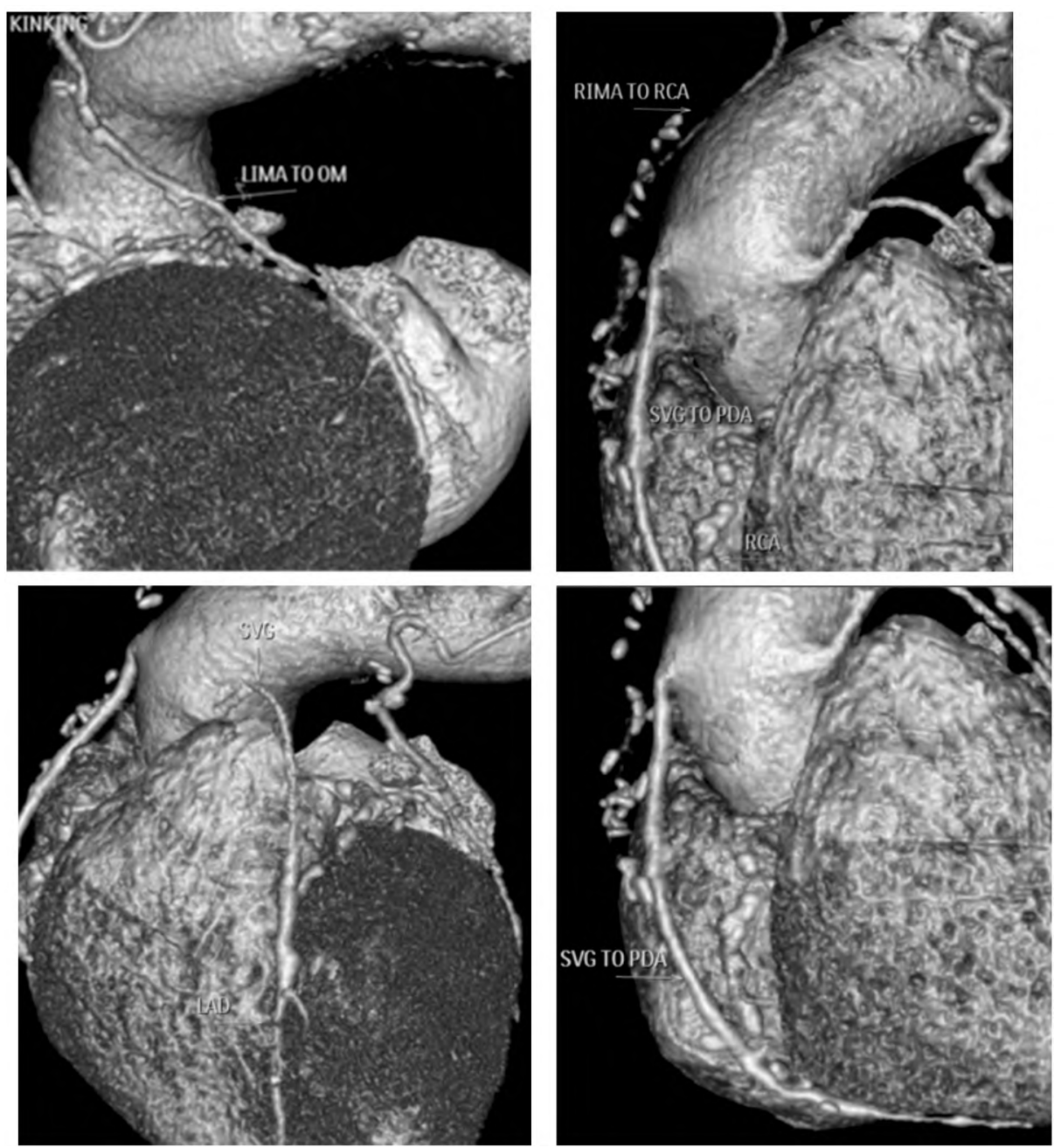

Fig. (2): 67 years old male patient, smoker and diabetic. In 2005, he developed recurrent attacks of chest pain. Diagnostic coronary angiography was done followed by a bypass operation. Five years later in 2010, pain recurred due to exaggerated native coronary arteries diseases so he underwent another CABG surgery. Chest pain recurred 1 month ago. 3D VR images shoving patent LIMA to OM with non-significant kinking, SVG1 to LAD and SVG2 to PDA with good distal contrast run off while RIMA to RCA is attenuated showing bad opacification at the site of anastomosis with weak distal flow of the native arteries. 

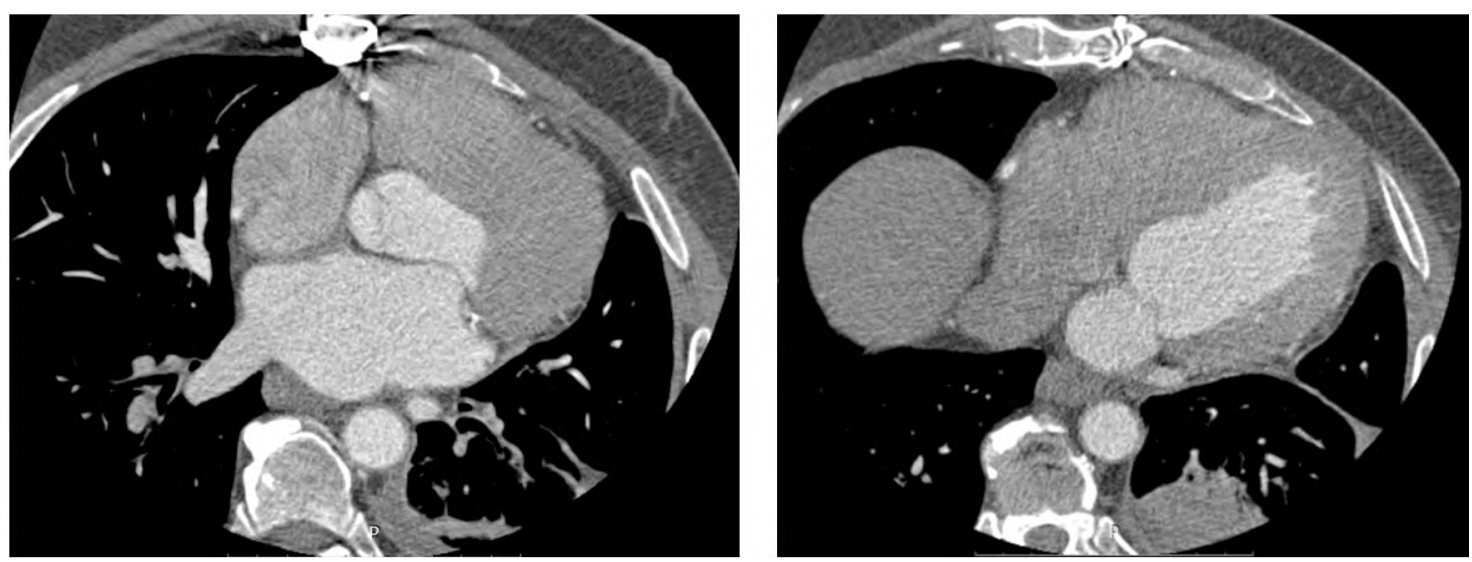

Fig. (3): A 55 years old male patient, smoker and not diabetic. In 2012, he developed recurrent attacks of chest pain followed by a bypass operation. Chest pain recurred 3 weeks ago, so he was referred for MDCT coronary angiography to evaluate the bypass grafts and to assess the condition of the native arteries. At time of examination he was suffered recently from fever and productive cough. Selected axial CT images reveal left pleural effusion and left lung lower lobe basal segments consolidations suggesting pneumonia.

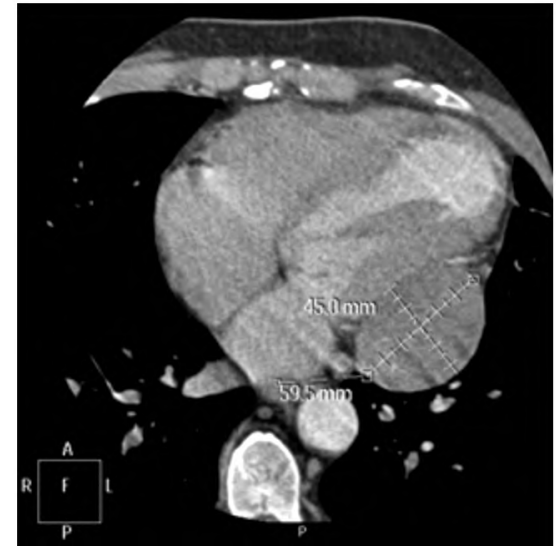

Fig. (4): A 48 years old male patient, smoker and non-diabetic. In 2014, he developed recurrent attacks of chest pain. Diagnostic coronary angiography was done followed by a bypass operation. Chest pain recurred 1 month ago, so he was referred for MSCT coronary angiography to evaluate the bypass grafts and to assess the condition of the native arteries. CT Axial cut at the level of the well-defined organized pericardial hematoma seen at left postero inferior aspect of the heart measuring $6 \times 4.5 \mathrm{~cm}$.
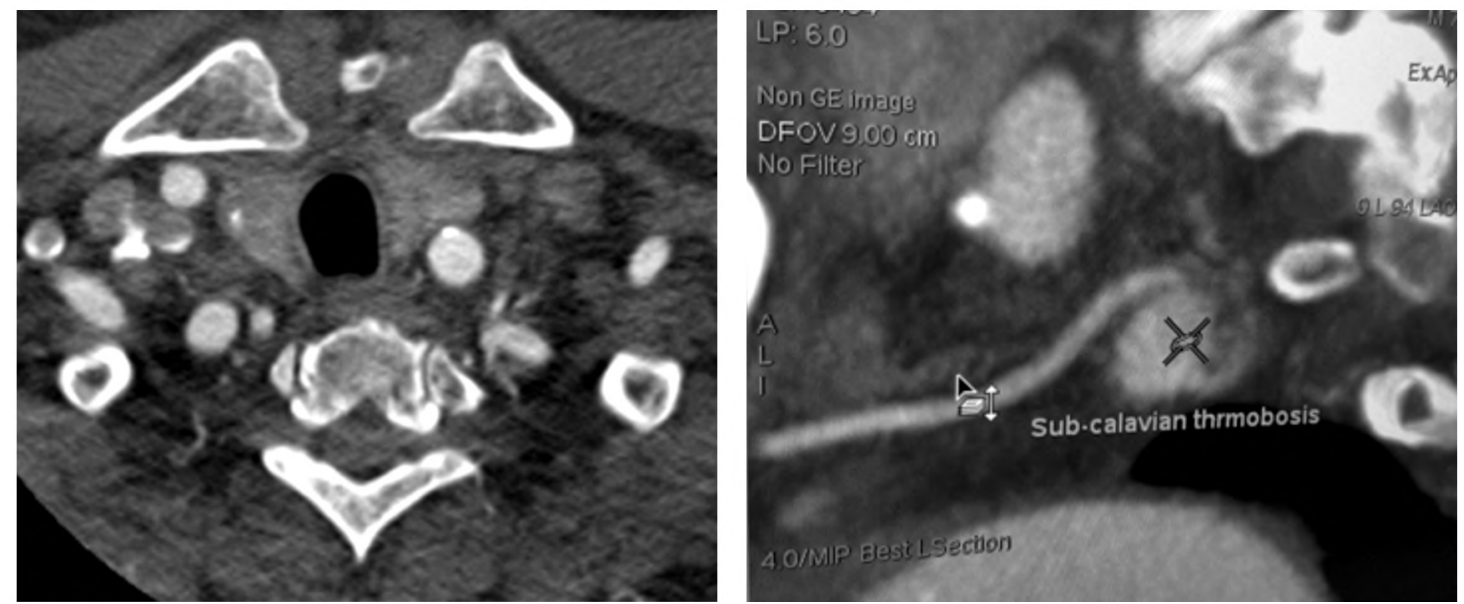

Fig. (6): 76 years old male patient, smoker and diabetic. In 2009, he developed recurrent attacks of chest pain. Diagnostic coronary angiography was done with followed by a bypass operation. Chest pain recurred 2 month ago. Axial and Curved MPR of the origin site of LIMA from the left SCA (subclavian artery) demonstrating the partial SCA thrombosis encroaching upon LIMA origin causing $80 \%$ stenosis. 


\section{Discussion}

This study was undertaken to form the basis of review on the role of MDCT in detecting post CABG complications. In our study all grafted and non-grafted vessels were visually classified as abnormal if they showed $>50 \%$ luminal narrowing and/or vessel occlusion. MDCTA was used as it is considered quick, reproducible, and less invasive than other procedures especially in high risk patients where the referring surgeons or treating physicians need clinical answers before treating the patient. One of the most important variables that determine the successful clinical outcome of coronary artery bypass graft placement is the shortand long-term patency rate of the grafts. For venous coronary artery bypass grafts, an occlusion rate of $15 \%-20 \%$ has been reported within the 1 st year after surgery. After 10 years post-surgery, the occlusion rate is 55-65\% according to Ascarelli et al., [8] which also state that patency rates at 10 years for the internal mammary artery graft are much higher than $90 \%$. Up to $10 \%$ of grafts become occluded during the perioperative period. After 10 years, occlusion rates of 59\% and $17 \%$ have been reported for venous and arterial grafts respectively. About $40 \%$ of patients who undergo bypass surgery will experience recurrence of symptoms within 6 years, and $25 \%$ of bypass grafts are found to be occluded at 5 years follow-up according to De Feyter et al., [9]. Venous grafts are still the most widely used type of grafts due to their abundancy. In our study also, venous grafts were the most common type of grafts. It represented 82 of the 129 grafts, nearly about $63.6 \%$. Yet, there is high rate of complications with venous grafts due to their high rate of occlusion and narrowing. Our study showed that $7.3 \%$ of the venous grafts were significantly narrowed and about $28.1 \%$ of them were totally occluded. This gives a rate of complications of about $35.4 \%$ knowing that our mean time after operation was about 6 years. The OM branch was the commonest site for venous graft landing $(24.4 \%)$ in our study followed by the RCA $(13.4 \%)$. Yet $(24.4 \%)$ of the venous grafts were occluded with no knowledge of their supposed landing site as they were only seen as a dimples or star like projections at their origin sites from the ascending aorta and due to lack of previous operative details. The in-situ arterial grafts are used more common than the free arterial grafts, as the later has more rates of complications. In our study $(4.3 \%)$ of the arterial grafts were free arterial grafts with no significant narrowing or occlusion in them. The radial artery is the most commonly used free arterial graft due to its easy harvesting. In our study, $50 \%$ of the free arterial grafts were radial artery graft. As for the in-situ arterial grafts, they are one of the most widely used grafts $(95.7 \%$ of the arterial grafts in our study), especially at the relatively younger patient due to their high patency rates after a long time, yet they are not abundant. The LIMA is the most widely used insitu arterial graft $(93.3 \%$ of the in-situ arterial grafts in our study). Due to its arterial nature, it has less incidence rate complications. The LIMA grafts showed a $92.8 \%$ rate of patency in our study which cope with a rate higher than $90 \%$ of patency after 10 years according to Ascarelli et al., [8] There was a $4.8 \%$ rate of significant narrowing and a $2.4 \%$ rate of occlusion in our study. The LAD is the most common site for landing of the LIMA graft, as it is the most important coronary artery branch supplying the left ventricle and due to other anatomical considerations as they are both anteriorly located in the chest. $88.1 \%$ of our LIMA grafts ended at the LAD.

So far, conventional coronary angiography has been considered the standard of reference for evaluation of the patency and luminal stenosis of coronary artery bypass grafts. However, the main drawbacks of conventional coronary angiography for this purpose include invasiveness, patient discomfort, and risk of complications. A less invasive imaging modality is desirable for evaluation of patients suspected of having graft stenosis or occlusion. Contrast-enhanced three-dimensional Magnetic Resonance (MR) angiography is a promising technique that allows both assessment of coronary artery bypass graft occlusion and graft stenosis with a sensitivity between $95 \%$ and $100 \%$ as Chiribiri et al., [9] stated. Limited spatial resolution and artifacts caused by vascular clips surrounding arterial grafts may limit MR angiography in the evaluation of small arterial bypass grafts, such as IMA bypass grafts. In addition, MR angiography cannot be performed in claustrophobic patients or in patients with cardiac pacemakers or defibrillators, which are often implanted in patients with coronary artery disease. Electron-beam CT has been demonstrated to be useful for evaluation of coronary artery bypass grafts, with a reported diagnostic accuracy of $90.1 \%$ for detection of graft patency according to Oudkerk et al., [10]. However, possible drawbacks of electron-beam CT include the limited volume coverage (maximum 40 sections acquired with a section thickness of $3 \mathrm{~mm}$, resulting in a maximal craniocaudal volume coverage of $12 \mathrm{~cm}$ ) and the fact that CT data can only be acquired with prospective ECG gating.

The electron beam CT scanner has no mechanically rotating components, and hence, the temporal 
resolution $(50-100 \mathrm{msec})$ is sufficiently high. However, the spatial resolution is lower than the MDCT. Electron beam CT is prospectively gated. When $1.5 \mathrm{~mm}$ slice thickness protocol is used, it can cover $6 \mathrm{~cm}$ in 40 cardiac beats, which allows only scanning of the proximal and middle coronary segments of a normal sized heart. The entire heart can be covered using the $3 \mathrm{~mm}$ protocol but at the expense of reduced spatial resolution.

The major improvements of the recently developed 64 section and dual source CT machines compared with the older scanners, include improved temporal resolution due to shorter gantry rotation time, better spatial resolution owing to submillimeter collimation, and considerably reduced scan acquisition times. Despite the recent technical advances, the 64-detector row CT coronary angiography is still sensitive to arrhythmia. Persistent irregular cardiac rhythm such as atrial fibrillation and frequent extra-systoles preclude MDCT coronary angiography. However, motion artifacts owing to mild arrhythmia (e.g. single ventricular extrasystole) can be diminished by manual repositioning the reconstruction windows.

The use of multi-detector row CT is gaining increasing acceptance for noninvasive cardiac imaging. Several studies have demonstrated successful application of multi-detector row CT angiography for assessment of coronary artery disease. In 2012, Abdelmonem et al., [1]. Reported their experience in the assessment of coronary artery bypass grafts by using ECG-gated multi-detector row CT angiography using a 64 detector row machine. In that study, the authors evaluated 24 patients with a total of 78 bypass grafts with conventional coronary angiography as the standard of reference. According to the findings of $\mathrm{Abd} \mathrm{El}$ Monem et al., [1] ECG-gated 64 MDCT angiography yielded an excellent diagnostic performance with regard to assessment of occlusion of the graft (100\% sensitivity \& $97.1 \%$ specificity). The major drawback of retrospectively ECG-gated multidetector row CT data acquisition is the radiation dose. Since the data are acquired with an overlapping helical pitch and continuous X-ray exposure, the applied radiation dose is higher than that in the prospectively ECG-triggered sequential acquisition. When compared with the mean effective dose values calculated for conventional coronary angiography, the mean effective dose with multidetector row CT angiography was higher by a factor of about five. However, by reducing the tube output during heart phases that are not likely to be targeted by the ECG-gated reconstruction (i.e., reconstruction intervals except 50\%-70\%) a dose reduction of up to $80 \%$ is possible according to Kobayashi et al., [11] . To have consistent data acquisition in our study, we did not vary the tube output at the different phases of the cardiac cycle.

Hamon et al., [12] made a study with their aim of work to perform a meta-analysis to evaluate the accuracy of 16-and 64-section spiral Computed Tomography (CT) to assess coronary artery bypass grafts. The results of their meta-analysis demonstrate that obstructive bypass graft disease can be detected by using at least a 16-section CT with a high diagnostic accuracy with a sensitivity of $98 \%$, a specificity of $97 \%$, with only $92.4 \%$ of scanned grafts being fully assessable. This performance is better than for non-graft coronary arteries as shown in a meta-analysis by Hamon et al., [13], on a pervessel basis where the sensitivity was $82 \%$ and specificity was $91 \%$, for the diagnosis of significant coronary artery stenosis. This improvement in performance may be explained by a number of differences between bypass grafts and native coronary arteries. Graft conduits often have a larger diameter, are less frequently calcified and are relatively immobile, making CT imaging less challenging. Moreover, they are more often occluded than stenosed, which may make the diagnosis of obstruction more clear cut. Indeed, in the studies where information about occlusion rate was given in addition to the overall rate of substantial stenosis, there was much better detection of occlusion than for substantial stenosis. The LIMA grafts are usually closely related to a lot of surrounding surgical clips that may make them non-evalubale. SVG are less susceptible to this problem, mainly due to their larger caliber and the less usage of surgical clips around them. Compared with conventional angiography, CT angiography is less costly, faster, does not require assembly of an angiographic team to perform the study, and is generally more available 24 hours a day. It permits a wider variety of manipulations of the volumetric data set for image display and analysis in contrast to the limited projections routinely obtained during conventional angiography, and has fewer potential complications. There are a number of other potential advantages for MDCT over coronary angiography in the assessment of CABG. MDCT is a less invasive technique than coronary angiography. In addition, coronary angiography for CABG is technically more difficult than for native vessels, with a longer duration of procedure, more contrast agent used, and an increased complication rate. With coronary angiography, it is sometimes difficult to locate the origin of the grafts and explore them selectively. In the case of patients who need repeat cardiac bypass surgery, MDCT offers the surgeon precise 
information about the position of the existing grafts and the existence of a calcification of the aorta. In patients who require additional aortic valve surgery, an assessment of aortic root, including size and calcification, is also possible.

In MDCT some of scanned grafts are not fully assessable and the reasons for non-assessability in their study are cardiac motion, respiratory artifacts, poor opacification, and the presence surgical clips. However, with a 64-detector row machine, with increased temporal and spatial resolution and therefore shorter acquisition time, there is an improvement in the rate of fully assessable scanned grafts compared with a 16-section scanner. Up to 40 seconds were needed for a complete assessment of CABG with a four-section scanner. This has been reduced to less than 30 seconds with a 16section scanner and to less than 16 seconds with the 64-section scanner. However, even with the latest scanners, surgical metal clips and evaluation of distal anastomoses remain challenging. Besides general limitations of cardiac MDCT (e.g. use of potentially nephrotoxic agents, radiation exposure, and necessary selection of patients, with an exclusion of patients with atrial fibrillation and severe respiratory disease), some limitations are specific to the evaluation of patients with CABG. First, data acquisition does not provide information about flow characteristics and the functional state of the graft. Second, surgical clips, severe calcifications and extensive atherosclerotic disease, frequently observed in patients with previous $\mathrm{CABG}$, impair the assessability of the native coronary segments as well as few percentages of their grafts. Third, the increased acquisition volume increases the radiation exposure, and the increased amount of contrast media that is required further increases the risk of nephrotoxicity. Unlike conventional angiography which has a role is limited to detect CABG complications the MDCT has the ability to detect also non-graft related complications and explain patient compliant as for example fever related to pneumonia or sternal infection. In study by Helmy et al., [14] state that; HD 64-slice MDCTA is non-invasive technique which shows to be effective in diagnosis most of the post-operative cardiothoracic complications and gaining more grants in difficult clinical situations.

Our study had one patient (2\%) show pleural effusion and pneumonia at the left side and complaint from chest pain, heaviness, productive cough and low grade fever. Jan et al., [15] mentioned that pleural effusion is common after CABG particularly one the left side and patient complaint from chest pain or heaviness, shortness of breath and hypoxia.
In our study three patients $(6 \%)$ complaint from fever one $(2 \%)$ due to pneumonia and two $(4 \%)$ due to chest wall infection. Khalpey et al., [16] reported that causes of fever in post open heart surgery is noninfectious causes include myocardial infarction, post pericardiotomy syndrome, and drug fever. Infectious causes include wound infection, urinary tract infection, pneumonia, catheter sepsis, and loculated areas of contaminated blood accumulation (e.g., pericardial and pleural). The two patients (4\%) whom show chest wall infection one $(2 \%)$ revealed chronic osteomyelitis and one $(2 \%)$ demonstrated deep sternal wound infection with retrosternal collection. In 2016 Meszaros et al., [17] stated that incidence of deep sternal wound infection varies from (0.4 to $4 \%)$.

Our study had 39 patients $(78 \%)$ were diabetic and according to Shirani and Soleymanzadeh [18] mentioned that obesity, diabetes and bilateral internal mammary grafting increase the risk of sternal infection. Also the study had 31 patients $(62 \%)$ were currently smokers. Khalpey et al., [16] reported that smokers patients have a higher incidence of pneumonia (15.3\% versus $3.6 \%$ in controls).

In our study there is one patient ( $2 \%$ ) show localized pericardial hematoma. Kapoor et al., [19] reported case of delayed complications related to retained pericardial pacing wires left in situ with consequent organized pericardial hematoma around wires presenting three months following aortic valve replacement surgery especially in patients on long term oral anticoagulants.

Our findings demonstrated 17 obese patients (34\%) had more than one complication. Demir et al., [20] mentioned that obesity does not increase short term mortality for open heart surgery, however, it increases the risk of post-operative pulmonary complications and discharge with morbidity.

One patient ( $2 \%)$ in our study show partial thrombosis of left subclavian artery encroaching upon the origin of in situ LIMA to LAD graft also the MDCT reveal in our study aneurysmal dilatation of ascending aorta and main pulmonary artery in 6 patients $(12 \%)$ and 2 patients $(4 \%)$ respectively as well as anomalous origin of RCA from Left Coronary Sinus (LCS) and LCA from NonCoronary Sinus (NCS) in one patient $(2 \%)$ respectively.

ECG gated cardiac 64 MDCT is invaluable following $\mathrm{CABG}$, detailed anatomical information of the heart chambers, pericardium and accurate dynamic evaluation of myocardial function, perfusion and integrity as well as noninvasiveness for 
diagnosis of post-operative cardiothoracic complications following open heart surgery and it is an excellent, quick, and safe techniques for followup. Future advances using 256, 320 and higher detector rows CT machines will open new frontiers in the assessment of the heart, coronary arteries and coronary grafts. Faster examinations with much less radiation dose are now possible. Further studies are needed to document the expected better imaging quality of these machines during the assessment of the native arteries as well as the coronary grafts. Also further studies are needed to compare between HD MDCT and other imaging modalities such as coronary angiography and/or Magnetic Resonance Imaging (MRI) and Magnetic Resonance Angiography (MRA) in detecting post-operative cardiothoracic complications following CABG surgery.

\section{Conclusion:}

64-slice MDCT is an easy non-invasive technique which showed to be effective in diagnosis of most post-operative.

CABG complications and is gaining more ground in difficult clinical situations.

\section{References}

1- ABDELMONEM S., NASR A.M.S., ALGEBALAY A.M.W., et al.: Sixty four multi-detectors CT coronary angiography: An easy and accurate method to detect graft patency post CABG. The Egyptian Journal of Radiology and Nuclear Medicine, 43: 377-81, 2012.

2- HELMY I.M., MOSTAFA M.M.S. and ARAFA O.E.: Diagnostic Performance of High definition 64-slice MDCTA in Post-Operative Cardiothoracic Complications in Patients after Open Heart Surgery. Journal of American Science, 10 (8), 2014

3- FRAZIER A.A., QURESHI F., READ K.M., ROBERT C. D.D.R., et al.: Coronary Artery Bypass Grafts: Assessment with Multidetector CT in the Early and Late Postoperative Settings, Radio-Graphics, 25: 881-96, 2005.

4- KARLO C.A., LESCHKA S., STOLZMANN P., et al.: A systematic approach for analysis, interpretation, and reporting of coronary CTA studies. Insights into imaging, 3: 215-28, 2012.

5- JUNGMANN F., MILDENBERGER P., EMRICH T., et al.: MDCT imaging of coronary artery bypass graftsUpdate. ECR 2014/10.1594/C-1594.

6- AVIRAM G., MOHR R., SHARONY R., et al.: Open heart reoperations after coronary artery bypass grafting: The role of preoperative imaging with multidetector computed tomography. Isr. Med. Assoc. J., 11 (8): 4659, 2009.
7- NAZERI I., SHAHABI P., TEHRAI, et al.: Assessment of Patients after Coronary Artery Bypass Grafting Using 64-Slice Computed Tomography. American Journal of Cardiology, 103: 667-73, 2009.

8- ASCARELLI A., FRANCONE M., CANNATA D., et al.: Role of multidetector CT in the evaluation of coronary artery bypass grafts. Imaging Med., 2 (1): 77-86, 2010.

9- CHIRIBIRI A., BOTNAR R.M. and NAGEL E.: Magnetic Resonance Coronary Angiography: Where Are We Today. Curr. Cardiol. Rep., 15 (2): 328, 2013.

10- OUDKERK M., REISER M.F., ACHENBACH S., et al.: Coronary Radiology. Medical Radiology: Diagnostic Imaging. Second revised edition. Springer Verlag Berlin Heidelberg, P. 154, 2009.

11-KOBAYASHI M., ASADA Y., MATSUBARA K., et al.: Electrocardiogram gated coronary $\mathrm{CT}$ angiography dose estimates using ImPACT. Journal of applied clinical medical physics, 17-4, 2016.

12- HAMON M., LEPAGE O., MALAGUTTI P., et al.: Diagnostic Performance of 16- and 64-Section Spiral CT for Coronary Artery Bypass Graft Assessment: MetaAnalysis. Radiology June, 247: 679-86, 2008.

13- HAMON M., BIONDI-ZOCCAI G., MALAGUTTI P., et al.: Diagnostic performance of multislice spiral computed tomography of coronary arteries as compared against conventional invasive coronary angiography: A metaanalysis. J. Am. Coll. Cardiol., 48: 1896-910, 2006.

14- HELMY I.M., ASBEUTAH A.M., ARAFA O.E. and ELFIKI I.M.: Pictorial Review on the Role of 64-Slice HDMDCT in Detecting Post CABG Cardiothoracic Complications. Open Journal of Thoracic Surgery, 4: 48-58, 2014.

15- JAN A., AKHTAR M.J., HAMEED K., et al.: Prevalence \& clinical outcome/course of pleural effusion in post CABG patients. The Journal of Cardiovascular Disease, 11-4, 2013.

16- KHALPEY Z.L., GANIM R.B. and RAWN J.D.: Postoperative care of cardiac surgery patients (chapter 16). Cardiac Surgery in the Adult textbook. Copyright in 2008, P. 479, 2008.

17- MESZAROS M., FUEHRER U., GROGG S., et al.: Risk Factors for Sternal Wound Infection After Open Heart Operations Vary According to Type of Operation. Ann. Thorac. Surg., 101: 1418-25, 2016.

18- SHIRANI S. and SOLEYMANZADEH M.: Spiral CT of Non-Graft Post Cardiac Surgery Complications: A Pictorial Essay. Iran J. Radiol., 7 (1): 11-5, 2010.

19-KAPOOR A., SYAL S., GUPTA N. and GUPTA A.: Right paracardiac mass due to organized pericardial hematoma around retained epicardial pacing wires following aortic valve replacement. Case report. Interactive Cardiovascular and Thoracic Surgery, 13: 104-6, 2011.

20- DEMIR A., AYDLNLL B., GUCLU C.Y., et al.: Obesity and post-operative early complications in open heart surgery. J. Anesth., Oct., 26 (5): 702-10, 2012. 


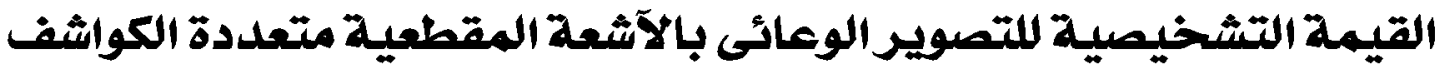

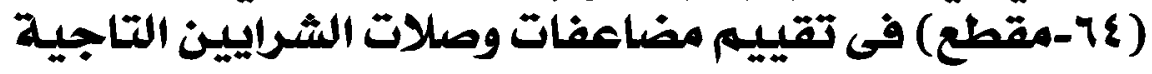

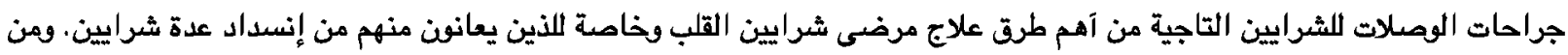

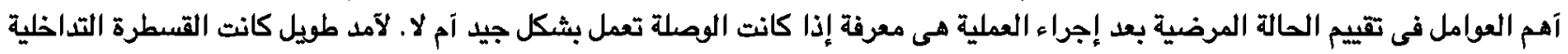

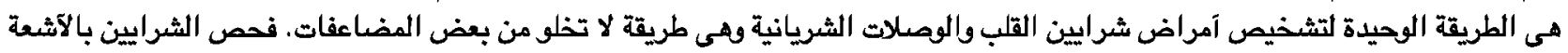

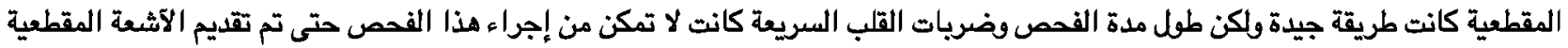

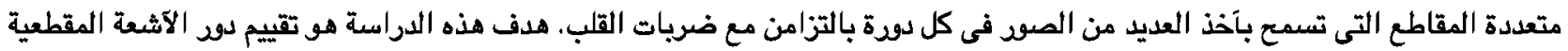

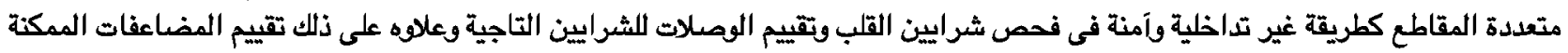

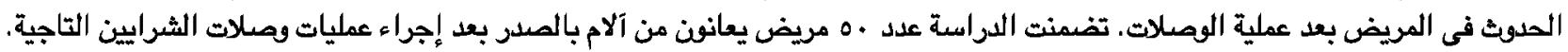

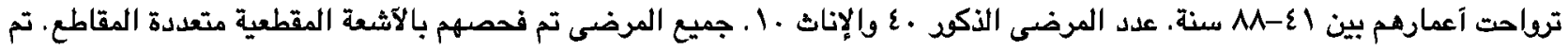

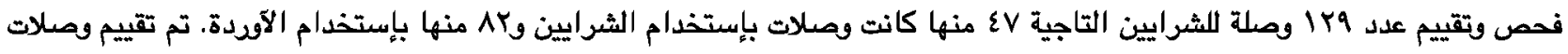

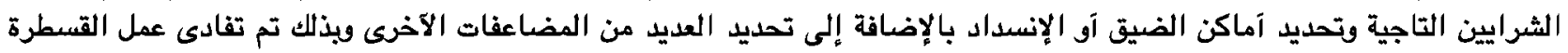

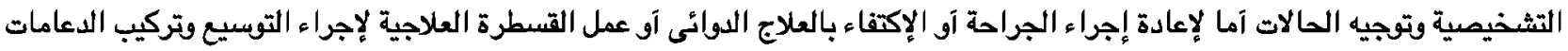

